\title{
Micromagnetic Analysis of Residual Stress Distribution in 42CrMo4 Steel after Thermal and Mechanical Surface Treatment
}

\author{
Ilya Bobrov ${ }^{1,2, a, *}$, Jérémy Epp ${ }^{1,3, b}$ and Hans-Werner Zoch ${ }^{1,2,3, c}$ \\ ${ }^{1}$ Universität Bremen, Badgasteiner Straße 1-3, 28359, Bremen, Germany \\ ${ }^{2}$ Leibniz-Institute for Materials Engineering - IWT, Badgasteiner Str. 3, 28359 Bremen, \\ Germany \\ ${ }^{3}$ MAPEX Center for Materials and Processing, University of Bremen, Germany \\ abobrov@iwt-bremen.de, bepp@iwt-bremen.de, 'zoch@iwt-bremen.de
}

Keywords: Residual Stresses, Micromagnetic, X-Ray Diffraction, Barkhausen Noise

\begin{abstract}
In this study, residual stresses and micromagnetic parameters were analyzed with a Barkhausen-Noise-Eddy-Current-Microscope which allows the analysis of local micromagnetic properties with high resolution down to $20 \mu \mathrm{m}$. 42CrMo4 steel samples with varying initial microstructure and subjected to different thermal and mechanical surface treatments were investigated. In particular, shot peening and induction hardening were used to generate different distributions of microstructural and residual stress modifications. Calibration strategies were developed using standard methods as X-ray diffraction measurements and metallographic examinations. The results show that a quantitative evaluation of residual stress distribution can be possible, even in regions with high gradients when proper measurement and calibration strategy is used. With this method, large areas with thousands of measurement positions can be analyzed very fast and thus open new possibilities in the investigation of local residual stress distribution of components.
\end{abstract}

\section{Introduction}

Thermal and mechanical surface treatments like shot peening, inductive hardening and their combinations induce local changes in the microstructural features such hardness or residual stress state of the material, while the level of modifications and the size of the affected region can be varied by the process parameters [1]. Induced microstructural changes can be detected and evaluated with well-known methods like X-Ray diffraction or hardness measurements [2]. These methods can provide information about the material state, which can be used to evaluate mechanical properties of the materials as well. On the other hand, fast non-destructive analysis like micromagnetic methods have high potential for large area investigation or high-throughput material screening since they are very sensitive to the microstructure and mechanical properties of the material [3]. However the data obtained with these methods cannot be directly used to describe material properties, but proper calibration is required. This calibration function can be obtained after suitable calibration and can be used to evaluate the mechanical properties [4]. The advantage of such methods is the non-destructivity and faster data collection as for the other methods.

In this study micromagnetic measurement were performed with Barkhausen Noise and Eddy Current Microscope based on the 3MA (Micromagnetic Multiparameter Microstructure and Stress Analysis) method from Fraunhofer Institut for Non-Destructive Testing, Saarbrücken, Germany. This method is described in details in [5].

In this equipment 3 different testing principles are realized and can be used to obtain data from small areas with high spatial resolution $(\sim 20 \mu \mathrm{m})[6]$ : Barkhausen Noise $(\mathrm{BN})$ analysis, 
incremental permeability analysis and Eddy Current (EC) method. The application of these methods with several testing frequencies provides more than 30 micromagnetic parameters, which correlate to the properties of the material structure and layered systems. The combination of the micromagnetic characteristics in a multiparameter approach enables the separation of overlapped influences, for example stress and layer thickness [7]. The 2D-maps of selected micromagnetic parameters can allow to gain information on material homogeneity and local property variations. Furthermore, the comparison and combination of the characteristics of several samples allows for a qualitative estimation of the differences in material properties [8].

In the present study $42 \mathrm{CrMo} 4$ steel samples were subjected to different surface treatments to induce varying microstructural and mechanical property distributions. Micromagnetic measurements were performed using the BEMI system, while X-ray diffraction analyses and hardness measurements were used to calibrate and interpret the micromagnetic parameters. The aim of the investigations was to achieve quantitative evaluation of local properties and to predict hardness and residual stress distributions in the different treatment conditions. Single micromagnetic parameters used in the function were evaluated individually to determine their sensitivity regarding the different target properties.

\section{Experimental methods}

The experiments were performed on samples from 42CrMo4 steel (AISI 4140H). The chemical composition of this steel contains $0.43 \%$ C, $0.26 \% \mathrm{Si}, 0.74 \% \mathrm{Mn}, 0.01 \% \mathrm{P},<0.001 \% \mathrm{~S}, 1.09 \%$ $\mathrm{Cr}$ and $0.25 \%$ Mo. For this study two initial material states with different hardness were prepared. The ferritic-pearlitic (FP) material state was achieved by soaking for $0.5 \mathrm{~h}$ at $840{ }^{\circ} \mathrm{C}$ and subsequent air cooling while a quenched and tempered (QT) state was achieved by quenching in $60^{\circ} \mathrm{C}$ oil after prior austenitizing with additional tempering at $450{ }^{\circ} \mathrm{C}$ for 1 hour.

X-ray diffraction was performed with MZ IV diffractometer of GE Inspection Technology, Ahrensburg, Germany with $\mathrm{Cr}-\mathrm{K}_{a 1,2}$ radiation, produced by long fine focus tube. The primary beam size was $200 \mu \mathrm{m}$ in diameter and was defined using a focusing mini-lens from IFG, Berlin, Germany. Diffracted signal was detected by position sensitive detector "Miostar 2" (Photron-X), equipped with vanadium filter. For residual stress, the $\{211\}$ peak of $\alpha$-Fe was measured at each position along $13 \chi$-angles between $-45^{\circ}$ and $+45^{\circ}$ and the residual stresses were calculated by standard $\sin ^{2} \psi$-method using X-ray elastic constants $1 / 2 \mathrm{~S}_{2}=5.8110^{-5} \mathrm{MPa}-1$ [9].

The basic part of the BEMI (Figure 1, A, B) is the 3-axes positioning system with a holder unit for the inductive probes and the reference Hall probe. The test specimen is fixed on an electromagnetic yoke with an additional Hall probe below the specimen. The magnetization unit consists of a function generator and an amplifier. This gives the ability to magnetize with a field strength of $0-125 \mathrm{~A} / \mathrm{cm}$ and frequencies up to $250 \mathrm{~Hz}$. The manipulation contro1 unit is a commercial system with an accuracy of $10 \mu \mathrm{m}$ in $\mathrm{x}, \mathrm{y}$, and $\mathrm{z}$ directions. The major parts of the microscope are the units of EC testing and BN measurement that can be se1ected by a software controlled switch. The EC unit allows simultaneous testing with four multiplexed frequencies between $10 \mathrm{~Hz}-10 \mathrm{MHz}$. The $\mathrm{BN}$ unit detects the induced $\mathrm{BN}$ in three filters ranges between 20 $\mathrm{kHz}$ and $10 \mathrm{MHz}$ with an amplification up to $100 \mathrm{~dB}$. The computer controls the system, the signal processing and stores the measured data [10].

In the present investigations, EC measurements were performed with 4 different exciting frequencies: $500 \mathrm{kHz} ; 1 \mathrm{MHz} ; 2 \mathrm{MHz} ; 3 \mathrm{MHz}$. Base magnetization frequency for the two other measurement methods was defined at $100 \mathrm{~Hz}$. BN analysis was performed using $25 \mathrm{~A} / \mathrm{cm}$ magnetization amplitude. Obtained signal was filtered with lowpass frequency of $100 \mathrm{kHz}$ and highpass frequency of $1 \mathrm{MHz}$. The magnetization amplitude for incremental permeability was chosen by $15 \mathrm{~A} / \mathrm{cm}$ and the EC loop frequency for this analysis was chosen by $1 \mathrm{MHz}$

The samples used for this study were $200 \times 30 \times 19 \mathrm{~mm}$ blocks of $42 \mathrm{CrMo} 4$ steels from which transversal cross section samples with $20 \times 20 \times 10 \mathrm{~mm}$ were prepared. Cross section surface was 
ground and polished. The treatment combination for the samples are: quenched and tempered with additional shot peening $(\mathrm{QT}+\mathrm{SP})$; quenched and tempered with additional induction hardening with surface hardening depth (SHD) of about $1 \mathrm{~mm}(\mathrm{QT}+\mathrm{IH})$; previous combination with additional shot peening $(\mathrm{QT}+\mathrm{IH}+\mathrm{SP})$; induction hardened sample with $\mathrm{SHD}=1 \mathrm{~mm}$, with ferritic-pearlitic initial microstructure $(\mathrm{FP}+\mathrm{IH})$. All measurements were performed in the area from the treated surface towards the sample center as shown in Figure 1C. Residual stress measurements were performed only for transversal stress component.
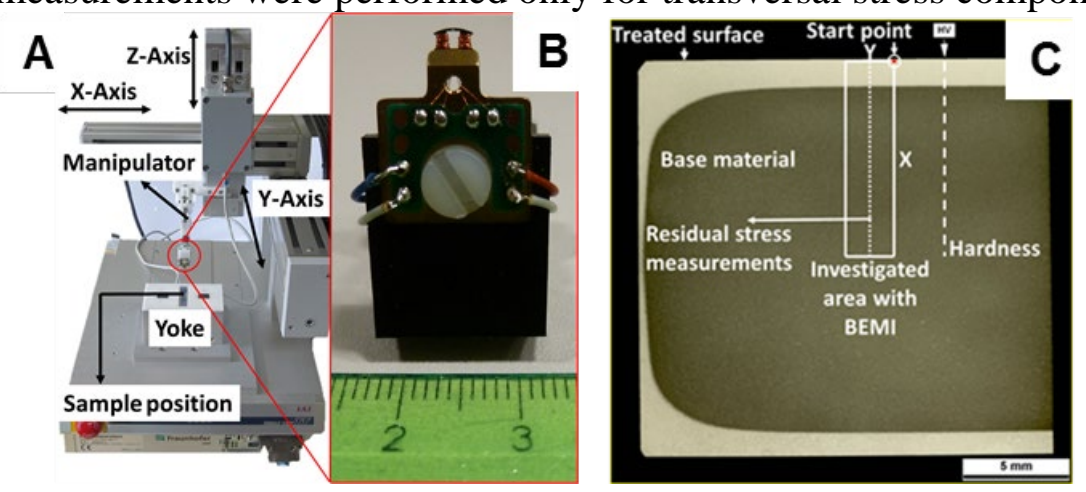

Fig 1. A) BEMI B) BEMISensor $C$ ) Investigated regions on the cross section of the sample

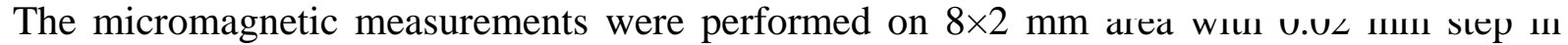
each direction (Figure 1, C). From the obtained 2D-maps, average of the 100 data points was calculated over the measured $2 \mathrm{~mm}$ in $\mathrm{y}$-direction for each $\mathrm{x}$-axis step, giving a mean parameter evolution from the surface to the depth.

\section{Results and discussion}

The results of hardness measurements, residual stress and two micromagnetic parameters for four different samples are shown in Figure 2.

After QT+SP, the hardness is almost constant around $420 \mathrm{HV} 1$ over the entire region, while the residual stress distribution exhibits increasing compression stresses towards the surface and slight compression at distances larger than $0.6 \mathrm{~mm}$ under the surface. In induction hardened state, the sample QT+IH has almost constant high hardness in the first $\mathrm{mm}$, followed by a decrease to minimum values at $1.3 \mathrm{~mm}$ depth slightly below the original hardness (about $80 \mathrm{HV} 1$ lower) which is then reached at $2 \mathrm{~mm}$ depth. This decrease is due to tempering effects below the hardened layer. Additional shot peening of sample QT+IH+SP leads to the same overall hardness distribution. Slight difference $(\sim 30 \mathrm{HV})$ between $\mathrm{QT}+\mathrm{IH}$ and $\mathrm{QT}+\mathrm{IH}+\mathrm{SP}$ samples in the first measured depth can be attributed to the shot peening process. In the case of sample $\mathrm{FP}+\mathrm{IH}$ similar high hardness is achieved in the first $0.8 \mathrm{~mm}$, followed by a decrease and almost constant value of $200 \mathrm{HV} 1$ below $1.3 \mathrm{~mm}$. On the other hand, it can be observed that the residual stress distributions of induction hardened samples with high compression in the hardened layer still present strong evolutions up to a depth of ca. $3 \mathrm{~mm}$. In particular, the QT+IH samples exhibit high tensile peaks. This different behavior of the properties can be used to separate both effects and calculate calibration functions on the base of micromagnetic parameters.

Distributions of maximum BN amplitude $\left(\mathrm{M}_{\max }\right)$ and of the coercivity value determined from Barkhausen peaks $\left(\mathrm{H}_{\mathrm{cm}}\right)$ are also shown in Figure $2(\mathrm{C}, \mathrm{D})$ over the distance from the treated surfaces for the 4 samples. Both micromagnetic parameters exhibit specific evolutions as a function of the distance from the surface, what is a consequence of the residual stress and hardness gradients in this region. For the QT+SP condition, only the surface near zone is affected by residual stress variations, while at depth higher than $0.6 \mathrm{~mm}$, more or less constant properties were detected. Nevertheless, the micromagnetic parameter exhibit linear evolution over the complete investigated depth which was not expected. Additional experiments with fully homogeneous samples showed, that this effect is the consequence of a magnetic field inhomogeneity within the sample. Strategies to eliminate or at least to compensate this 
instrumental influence will have to be developed in further investigations in order to increase the reliability of these analyses. The three samples in IH condition exhibit comparable evolution of $\mathrm{M}_{\max }$ with low values at the surface, followed by increasing tendencies. The two QT+IH samples have maximum values around $2 \mathrm{~mm}$ below the surface which decrease again towards the core, while the $\mathrm{FP}+\mathrm{IH}$ sample shows only a continuous increase over the depth. The distribution of $\mathrm{M}_{\max }$ for $\mathrm{QT}+\mathrm{IH}+\mathrm{SP}$ sample has a local maximum on the $0-0.6 \mathrm{~mm}$ distance from the surface. Finally, it can be observed that the $\mathrm{M}_{\max }$ curves converge to comparable values at highest depth for all samples. These distributions are very similar to the residual stress curves for each of the samples. On the other hand, $\mathrm{H}_{\mathrm{cm}}$ values exhibit different characteristics. The $\mathrm{H}_{\mathrm{cm}}$ values distribution for inductive hardened samples with QT initial state can be divided into 3 regions. First constant or only slightly increasing values from the surface to a depth around $1.3 \mathrm{~mm}$, where it then starts to decrease. This correlates well with the SHD of the samples. Then $\mathrm{H}_{\mathrm{cm}}$ is decreasing continuously up to the $2 \mathrm{~mm}$ and stabilizes at constant value below $3 \mathrm{~mm}$. This further evolution is not as sharp as the hardness profile what indicates that both hardness and residual stresses influence this parameter. In the case of QT+IH+SP, a slight increase of $\mathrm{H}_{\mathrm{cm}}$ occurs close to the treated surface, what is a consequence of the shot peening. The sample with the FP initial state has has a maximum of $\mathrm{H}_{\mathrm{cm}}$ values by the depth of $1.3 \mathrm{~mm}$ followed by a slow continuous decrease and ends at much lower value than the QT initial microstructure. This behavior also reflects the combination of hardness and residual stress influences for the FP initial microstructural state.
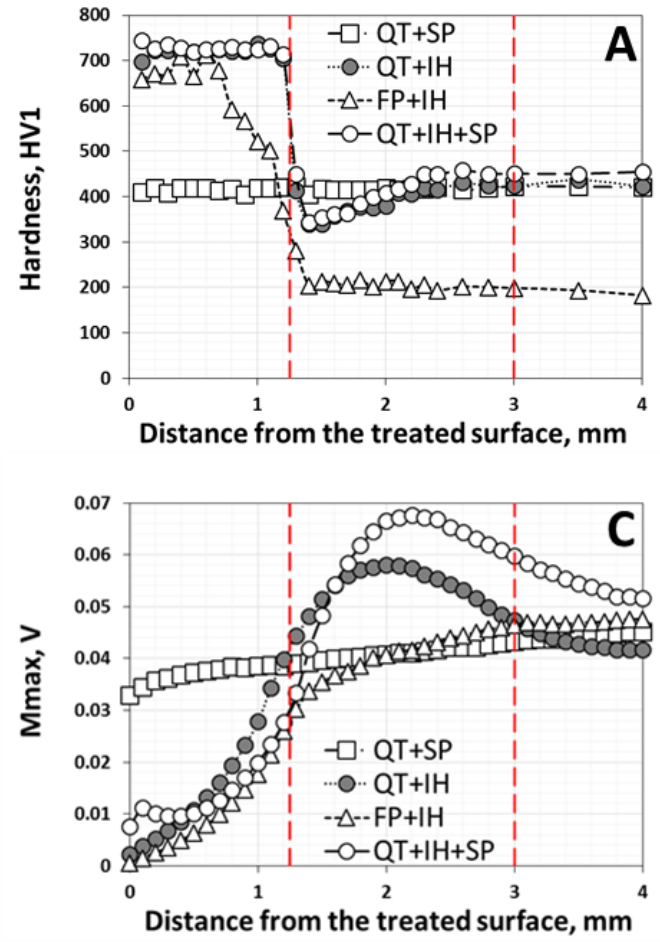
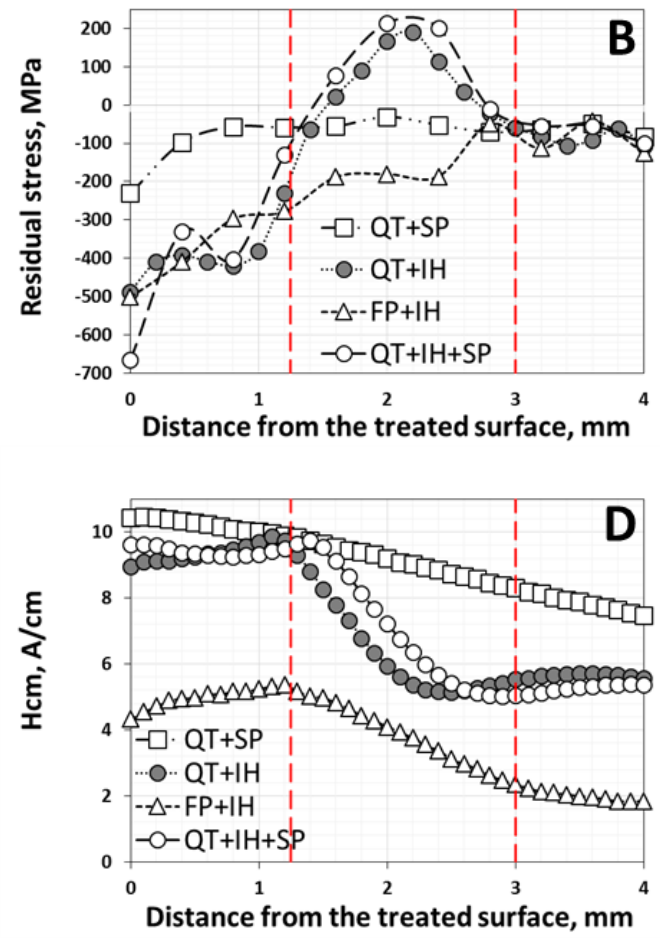

Fig. 2. Average evolution of properties for 4 different surface treatment combinations (QT= Quenched and Tempered;

IH=Induction Hardening; FP= Ferrite-Pearlite; $S P=$ Shot Peened): A) Hardness; B) Residual stresses; C) Maximum $B N$ amplitude $\left(M_{\max }\right)$; D) Coercivity value from $B N$ analysis at $M(H)=M_{\max }$.

The results were then used to determine suitable calibrations. Preliminary studies showed small difference in $r^{2}$-factors using more than 6 coefficients. Due this fact the calibration functions were obtained using regression analysis with 5 coefficients allowed. To achieve that, the reference points were chosen close to real X-Ray spot position with the averaging radius of $200 \mu \mathrm{m}$. Calibration was performed using all reference points for all investigated samples at the same time with the tool implemented in the 3MA software. Calibration functions were determined to analyze the hardness and residual stress distribution over the investigated area.

The following calibration functions were obtained: 
$H V=7.43 \times 10^{2}-3.24 \times 10^{4} \times M_{\text {mean }}+7.13 \times 10^{4} \times M_{\text {max }}{ }^{2}-1.99 \times 10^{3} \times \sqrt{\left|M_{\text {mean }}\right|}-$ $9.69 \times 10^{2} \times \sqrt{\left|M_{r}\right|}$.

$R S=-5.04 \times 10^{2}+1.26 \times 10^{5} \times M_{\text {max }}^{2}+6.96 \times 10^{1} \times \sqrt{\left|H_{c m}\right|}+1.77 \times 10^{-1} \times$ DH50m $\mathrm{m}^{2}-6.49 \times 10^{3} \times \mathrm{Im}_{4}{ }^{2}$.

Here, HV is the hardness, RS is the transversal residual stress value, $M_{\text {mean }}$ is the time averaged value of $\mathrm{M}(\mathrm{H})$-curve from $\mathrm{BN}$ analysis over 1 period, $M_{\max }$ is the maximum amplitude of $\mathrm{BN}, M_{r}$ is the $\mathrm{M}(\mathrm{H})$ value by $\mathrm{H}=0, H_{c m}$ is coercive field strength from $\mathrm{BN}$ analysis measured from $\mathrm{M}(\mathrm{H})$ curve by $\mathrm{M}=M_{\max }$, DH50m is the BN peak broadening by $\mathrm{M}(\mathrm{H})=0,5 \times M_{\max }$ and $\mathrm{Im}_{4}$ is the imaginary part of the impedance for highest frequency of the EC test.

These calibration functions were then used to calculate hardness and residual stress distributions from the micromagnetic investigations. The results are shown in Figure 3.
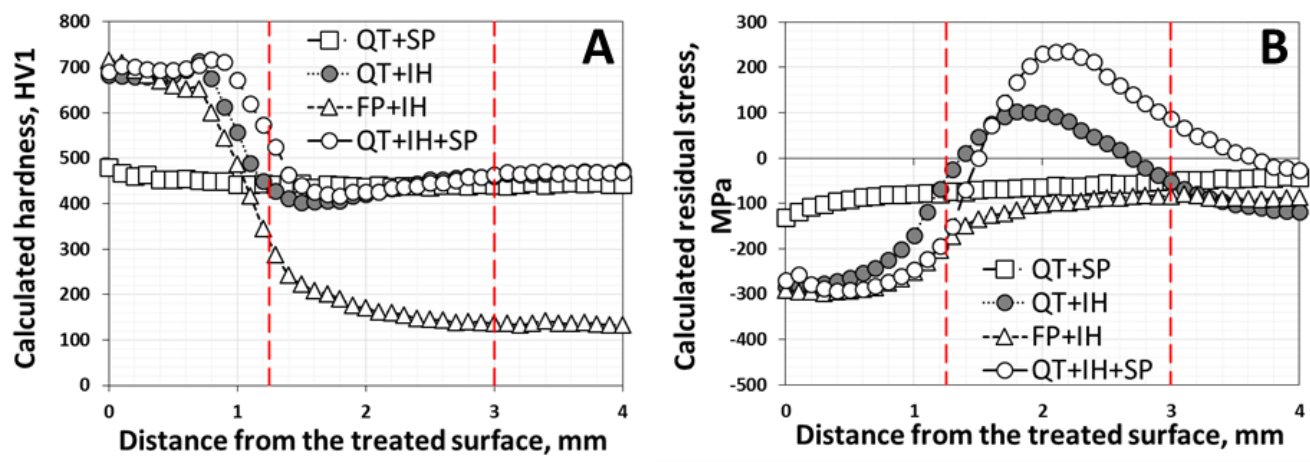

Fig. 3. Average evolution of calculated properties for the 4 different surface treatment combinations $(Q T=$ Quenched and Tempered; $I H=I n d u c t i o n$ Hardening; FP= Ferrite-Pearlite; $S P=$ Shot Peened): A) Hardness; B) Residual stresses;

Calculated hardness distributions show good agreement with the measured hardness curves. The deviations from the measured hardness can be divided into 2 groups. First are the small deviations: ca. $50 \mathrm{HV}$ difference for the QT+SP sample and also for the hardness close to the surface and of the base material of the IH samples. Larger deviations reaching $100 \mathrm{HV}$ are resulting for base material hardness of FP sample. In this case, the calculated depth evolution is not as sharp as the measured values and a more progressive change is calculated over the depth. This can be explained by the fact that there is no micromagnetic values, which have sharp evolutions as the hardness curve. Indeed, hardness is a complex property of several microstructural features, and therefore several contributions can be expected on the micromagnetic parameters. By increasing the number of terms in the calibration function the reliability of calculated hardness can be further increased. Calculated residual stress distribution exhibit larger deviations from the measured curves. In particular, the surface near high compression stresses cannot be predicted reliably, while tensile peaks in QT+IH samples and low compression towards the core are close to the measured values for all samples. This can be explained by the saturation behavior of several micromagnetic parameters in compression range, while high sensitivity is achieved in tensile regime [11].

From the obtained results, it could be shown that measurements and calibration of micromagnetic scans using BEMI is capable to achieve quantitative analyses of residual stresses and hardness distributions in surface treated samples, but optimization of the procedure is still required to improve the reliability of the predicted values.

\section{Conclusions}

In the present study, steel grade $42 \mathrm{CrMo} 4$ was produced in two different heat treatment conditions and submitted to thermal and mechanical surface hardening treatments. Various 
distributions of residual stresses and hardness were generated over the depth. By using a microscale micromagnetic measurements, evolution of micromagnetic parameters were determined in complete areas with high spatial resolution, and compared to the material properties measured by standard methods. As already known for macroscopic micromagnetic analysis the parameters can have different sensibility to the different microstructural features and properties.

It was shown, that $\mathrm{BN}$ analysis is very sensitive to hardness and residual stresses. Calibration function obtained for the hardness distribution contains only the values from BN analysis, when the calibration function is restricted to contain only 5 micromagnetic values. $\mathrm{M}_{\max }$ values distributions are more representative for residual stress distribution but the calculation requires more parameter. Further investigations are necessary to obtain reliable calibration function using this results. On the other hand there $\mathrm{H}_{\mathrm{cm}}$ values from the same analysis method are influenced by both hardness and residual stresses. For improvement of calculation reliability, more parameters should be included into the calibration function.

From the obtained results, it could be shown that the calibration of the system can allow the quantitative analyses of residual stresses and hardness distributions in surface treated samples with high spatial resolution, but optimization of the procedure is still required to improve the reliability of the predicted values. In particular, the effect of non-homogeneous magnetization should be taken into account. This factor is particularly relevant for larger samples dimensions. For the elimination of this effect a correction/compensation procedure is required, which is currently under development. Finally, multiple-objective optimization methods might be applied to take the single contributions and their overlapping into account.

\section{Acknowledgments}

Financial support of subproject D01 "Qualification of material conditions with mechanical and physical measuring methods" within the Collaborative Research Center SFB 1232 "Farbige Zustaende” by the German Research Foundation (DFG) is gratefully acknowledged.

\section{References}

[1] Schulze, Volker. Modern mechanical surface treatment: states, stability, effects. John Wiley \& Sons, 2006.

[2] J. Epp, XRD methods for materials Characterization in Material Characterization Using Nondestructive

Evaluation (NDE) Methods, Woodhead publishing pp. 81-124 (2016) https://doi.org/10.1016/B978-0-08-100040-

3.00004-3

[3] Blitz, Jack. Electrical and magnetic methods of non-destructive testing. Vol. 3. Springer Science \& Business Media, 2012.

[4] J. Epp, T. Hirsch: Residual Stress State Characterization of Machined Components by X-ray Diffraction and Multiparameter Micromagnetic Methods, Experimental Mechanics 50 (2010) 1, 195-204

[5] I. Altpeter, et al., Electromagnetic and Micro-Magnetic Non-Destructive Characterization (NDC) for Material Mechanical Property Determination and Prediction in Steel Industry and in Lifetime Extension Strategies of NPP Steel Components, Inverse Problems, 18 (2002) 1907 -1921. https://doi.org/10.1088/0266-5611/18/6/328

[6] K. Szielasko, et al., Ortsauflösende Charakterisierung ferro- und ferrimagnetischer Schichten für magnetoresistive und magnetooptische Sensoren. GZfP-Jahrestagung 2014 - Di.1.B.4

[7] M. Abuhamad, I. Altpeter, G. Dobmann, M. Kopp, Non-destructive characterization of cast iron gradient combustion engine cylinder crankcase by electromagnetic techniques (in German), Proceedings of the DGZfPAnnual Assembly (2007), Fürth

[8] B.Wolter, G. Dobmann, Micromagnetic Testing for Rolled Steel, European Conference on Non-destructive Testing (9) (2006) Th. 3.7.1, 25.-29. 09. 2006, Berlin.

[9] Noyan, Ismail C., and Jerome B. Cohen. Residual stress: measurement by diffraction and interpretation. Springer, 2013.

[10] Bender, J., D. O. Thompson, and D. E. Chimenti. "Barkhausen Noise and Eddy Current Microscopy (BEMI): Microscope Configuration, Probes and Imaging Characteristics in 'Review of Progress in Quantitative Nondestructive Evaluation." (1997): 212Iff.

[11] Stewart, D. M., K. J. Stevens, and A. B. Kaiser. "Magnetic Barkhausen noise analysis of stress in steel." Current Applied Physics 4.2-4 (2004): 308-311. 